\title{
Determining the Solar Inactivation Rate of BK Polyomavirus by Molecular Beacon
}

\author{
Dane C. Reano* and Marylynn V. Yates \\ Department of Environmental Sciences, University of California, Riverside, CA 92521, USA
}

Supporting information

7 Pages, 1 Table (BKPyV Alignments), 2 Figures (Solar Output)

*Phone: +1-951-827-4305. E-mail: drean001@ucr.edu 


\section{Media and buffer used for propagation of renal proximal tubule cells:}

Preformulated renal epithelial medium (REM) used for the propagation of primary renal proximal tubule cells (RPTEC) was obtained from Lonza (Basel, Switzerland). The proprietary basal media and necessary growth factors were ordered together (REGM BulletKit, CC-3190, Lonza). The utilized growth factors for a $500 \mathrm{~mL}$ batch of REM are as follows: $0.5 \mathrm{~mL}$ human epidermal growth factor, $0.5 \mathrm{~mL}$ hydrocortisone, $0.5 \mathrm{~mL}$ epinephrine, $0.5 \mathrm{~mL}$ insulin, $0.5 \mathrm{~mL}$ triiodothyronine, $0.5 \mathrm{~mL}$ transferrin, $1000 \mathrm{U}$ gentamicin and amphotericin, and varied amounts of heat inactivated fetal bovine serum (FBS).

The concentrations of FBS depended on the experiments performed; specifically, cell growth 10\%, BK Polyomavirus (BKPyV) propagation 5\%, and immunofluorescence and molecular beacon assays $2 \%$. The size and sensitivity of growth factors precluded sterilization of media; therefore, heat inactivation of FBS was necessary. Polyomaviruses are common contaminants of FBS $^{1}$ and cytopathic effect was even seen in initial experiments utilizing untreated FBS (data not shown). To this end, FBS provided from the manufacturer was heat inactivated at $56{ }^{\circ} \mathrm{C}$ for one hour with gentle mixing for the first 5 minutes, then kept at $4{ }^{\circ} \mathrm{C}$ until same day use.

The RPTEC were washed with HEPES buffered saline (HBS). A formulation for a $2 \times \mathrm{HBS}$ is as follows: $50 \mathrm{mM}$ HEPES, $274 \mathrm{mM} \mathrm{NaCl}, 10 \mathrm{mM} \mathrm{KCl}, 1.48 \mathrm{mM} \mathrm{Na}_{2} \mathrm{HPO}_{4}$, and $11.1 \mathrm{mM}$ dextrose. The $2 \times$ solution was adjusted to a $\mathrm{pH}$ of 7.05 with $10 \mathrm{~N} \mathrm{NaoH}, 0.22 \mu \mathrm{m}$ filter sterilized, and kept at $4{ }^{\circ} \mathrm{C}$. Finally, the $2 \times \mathrm{HBS}$ was diluted to $1 \times$ with $\mathrm{H}_{2} \mathrm{O}$, adjusted to $\mathrm{pH}$ of 7.3 with $2 \mathrm{~N} \mathrm{NaoH}, 0.22 \mu \mathrm{m}$ filter sterilized, and stored at $4{ }^{\circ} \mathrm{C}$.

\section{Immunofluorescence Assay:}


A protocol from Moriyama and Sorokin (2008) served as a general guide for the immunofluorescence assay (IFA) utilized in this study. Specifically, RPTEC were split into Nunc ${ }^{\circledR}$ Lab-Tek 8 well Permanox ${ }^{\circledR}$ chamber slides $\left(0.8 \mathrm{~cm}^{2}\right.$ per well, C7182-1, Sigma-Aldrich, St. Louis, MO, USA) at a concentration of 1E5 cells per well and incubated overnight at $37{ }^{\circ} \mathrm{C}$ with $5 \% \mathrm{CO}_{2}$. Wells that were $80 \%$ confluent were infected with $150 \mu \mathrm{L}$ of viral inoculum diluted in REM $2 \%$ FBS. The inoculum was aspirated after one hour of incubation with gentle rocking every 15 minutes and replaced with $300 \mu \mathrm{L}$ REM $2 \%$ FBS and allowed to incubate for 72 hours.

After incubation, wells were washed with $350 \mu \mathrm{L}$ of autoclaved Tris Buffered Saline (TBS, $150 \mathrm{mM} \mathrm{NaCl}, 20 \mathrm{mM}$ Tris base, $\mathrm{pH}$ 7.5) and fixed with $150 \mu \mathrm{L}$ ice cold absolute methanol for 20 minutes at $-20{ }^{\circ} \mathrm{C}$. Cells were then permeabilized with $300 \mu \mathrm{L}$ TBS containing $0.1 \%(\mathrm{v} / \mathrm{v})$ Tween $^{\circledR} 20(\mathrm{TTBS})$ and $1 \%(\mathrm{w} / \mathrm{v})$ bovine serum albumin fraction V (BSA) for five minutes. The TTBS 1\% BSA was then aspirated and replaced with $300 \mu \mathrm{L}$ TTBS $3 \%$ BSA and allowed to incubate at room temperature for 30 minutes to block nonspecific antibody binding. After blocking, wells were aspirated and exposed to a primary antibody from mice specific for the T antigen of the closely related SV40 Polyomavirus (PAb416, EMD Millipore, Billerica, MA, USA). Specifically, $150 \mu \mathrm{L}$ of the primary IgG antibody, diluted to $1 \mu \mathrm{g} / \mathrm{mL}$ in TTBS $1 \%$ BSA, was added to each well and allowed to incubate at room temperature for 1 hour. Wells were then washed with $350 \mu \mathrm{L}$ of TTBS $1 \%$ BSA for 5 minutes at room temperature to remove unbound antibodies. Antibody binding was detected through the addition of a fluorescein conjugated secondary anti-mouse IgG antibody grown in goats (DP02, EMD Millipore). To this end, we added $150 \mu \mathrm{L}$ of the secondary antibody diluted to $5 \mu \mathrm{g} / \mathrm{mL}$ in TTBS $1 \%$ BSA, and 
allowed to incubate for 40 minutes at room temperature. Unbound secondary antibodies were removed by washing wells with $350 \mu \mathrm{L}$ of TTBS $1 \%$ BSA for 5 minutes at room temperature.

Finally, wells were stained and mounted with VECTASHIELD ${ }^{\circledR}$ Mounting Media with DAPI (1.5 $\mu \mathrm{g} / \mathrm{mL}, \mathrm{H}-1200$, Vector Laboratories, Burlingame, CA, USA) before imaging on an IX71 inverted fluorescent microscope utilizing a 100W Hg bulb burner (U-RFL-T, Olympus, Tokyo, Japan) and a Retiga EXi Fast 1394 CCD camera (QImaging, Surrey, BC, Canada). All filters used in experiments were obtained from Chroma Technology Corporation (Bellows Falls, VT, USA); specifically, DAPI (31000v2; AT350/50x;400dclp;D460/50m) and fluorescein (49002; ET470/40x;T495lpxr;ET525/50m). Finally, all images were processed with Image-Pro version 6.3 (Media Cybernetics, Incorporated, Rockville, MD, USA).

\section{Sequences used in designing the BKPyV Molecular Beacon:}

Conserved sequences of BKPyV for molecular beacon design were identified through aligning 30 sequences in Clustal Omega (EMBL-EBI, Cambridgeshire, UK). 


\begin{tabular}{|c|c|c|c|}
\hline $\begin{array}{l}\text { Accession } \\
\text { Number }\end{array}$ & $\begin{array}{c}\text { Position } \\
\text { Start }\end{array}$ & Sequence & $\begin{array}{c}\text { Position } \\
\text { End }\end{array}$ \\
\hline 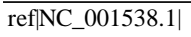 & 1646 & T A A T A A A A G G A G G A G T A G A A G T T C T A G A A T T A A A A C T G G G T A G A T G C T T & 1697 \\
\hline $\mathrm{dbj}|\mathrm{AB} 485709.1|$ & 1561 & T A A $T$ A A A A G G A G G A G T A G A A T T C T A G A A T T A A A A C T G G G T A G A T C T A T & 1612 \\
\hline dbj|AB298942.1| & 1526 & T A A T A A A A G G A G G A G T A G A A G T T C T A G A A T T A A A A C T G G G T A G A T G C T & 1577 \\
\hline $\mathrm{dbj}|\mathrm{AB} 369095.1|$ & 1526 & T A A T A A A A G G A G G A G T A G A A G T T C T A G A A T T A A A A C T G G G T A G A T G T A T & 1577 \\
\hline $\mathrm{dbj}|\mathrm{AB} 365170.1|$ & 1526 & T A A T A A A A G G A G A G T A G A A G T T C T A G A A T T A A A A C T G G G T A G A T G C T A T & 1577 \\
\hline $\mathrm{dbj}|\mathrm{AB} 217921.1|$ & 1526 & T A A T A A A A G G A G A G T A G A A G T T C T A G A A T T A A A A C T G G G T A G A T G T A T & 1577 \\
\hline $\mathrm{dbj}|\mathrm{AB} 464954.1|$ & 1526 & T A A T A A A A G G A G G A G T A G A A G T T C T A G A A T T A A A A C T G G G T A G A T G T A T & 1577 \\
\hline $\mathrm{dbj}|\mathrm{AB} 298946.1|$ & 1526 & T A A $T$ A A A A G G A G G A G T A G A A G T T C T A G A A T T A A A A C T G G G T A G A T C T A T & 1577 \\
\hline dbj|AB298944.1| & 1526 & T A A T A A A A G G A G G A G T A G A A G T T C T A G A A T T A A A A C T G G G T A G A T C T A T & 1577 \\
\hline dbj|AB298940.1| & 1526 & T A A T A A A A G G A G G A G T A G A A G T T C T A G A A T T A A A A C T G G G T A G A T C T A T & 1577 \\
\hline dbj|AB485694.1| & 1526 & T A A T A A A A G G A G G A G T A G A A G T T C T A G A A T T A A A A C T G G G T A G A T G T A T & 1577 \\
\hline dbj|AB298941.1| & 1526 & T A A T A A A A G G A G G A G T A G A A G T T C T A G A A T T A A A A C T G G G G T A & 1577 \\
\hline dbj|AB263926.1 & 1526 & T A A T A A A A G G A G G A G T A G A A G T T C T A G A A T T A A A A C T G G G T A G A T G T A T & 1577 \\
\hline $\mathrm{dbj}|\mathrm{AB} 263912.1|$ & 1526 & T A A T A A A A G G A G G A G T A G A A G T T C T A G A A & 1577 \\
\hline gb|JF894228.1| & 1665 & T A A T A A A A G G A G G A G T A G A A G T T C T A G A A T T A A A A C T G G G T A G A T C T A T & 1716 \\
\hline dbj|AB369090.1| & 1526 & T A A T A A A A G G A G G A G T A G A A G T T C T A G A A T T A A A A C T G G G T A G A T G T A T & 1577 \\
\hline $\mathrm{dbj}|\mathrm{AB} 301090.1|$ & 1526 & T A A T A A A A G G A G A G T A G A A G T T C T A G A A T T A A A A C T G G G T A G A T G C T A T & 1577 \\
\hline $\mathrm{gb}|\mathrm{JN} 192431.1|$ & 1634 & T A A T A A A A G G A G G A G T A G A A G T T C T A G A A T T A A A A C T G G G T A G A T G C T A T & 1685 \\
\hline $\mathrm{gb}|\mathrm{DQ} 989801.1|$ & 1259 & T A A T A A A A G G A G G A G T A G A A G T T C T A G A A T T A A A A C T G G G T A G A T G T A T & 1310 \\
\hline $\mathrm{gb}|\mathrm{DQ} 989795.1|$ & 1259 & T A A T A A A A G G A G G A G T A G A A G T T C T A G A A T T A A A A C T G G G C T A A T G C T A T & 1310 \\
\hline dbj|AB263915.1| & 1526 & T A A T A A A A G G A G G A G T A G A A G T T C T A G A A T T A A A A C T G G G T A G A T G T A T & 1577 \\
\hline $\mathrm{dbj}|\mathrm{AB} 263925.1|$ & 1517 & T A A T A A A A G G A G G A G T A G A A G T T C T A G A A T T A A A A C T G G G T A G A T G $\mathrm{T}$ A T & 1568 \\
\hline $\mathrm{dbj}|\mathrm{AB} 260031.1|$ & 1526 & T A A T A A A A G G A G G A G T A G A A G T T C T A G A A T T A A A A C T G G G T A G A T G T A T & 1577 \\
\hline $\mathrm{dbj}|\mathrm{AB} 369087.1|$ & 1526 & T A A T A A A A G G A G G A G T A G A A G T T C T A G A A T T A A A A C T G G G T A G A T G T A T & 1577 \\
\hline emb|FR720314.1| & 1526 & T A A T A A A A G G A G G A G T A G A A G T T C T A G A A T T A A A A C T G G G T A G A T G $\mathrm{T}$ A T & 1577 \\
\hline gb|DQ989799.1| & 1259 & T A A T A A A A G G A G G A G T A G A A G T T C T A G A A T T A A A A C T G G G T A G A T G $\mathrm{T}$ A T & 1310 \\
\hline $\mathrm{dbj}|\mathrm{AB} 269840.1|$ & 1527 & T A A T A A A A G G A G G A G T A G A A T T C T A G A A T T A A A A C T G G G T A G A T C T A T & 1578 \\
\hline $\mathrm{dbj}|\mathrm{AB} 263916.1|$ & 1525 & T A A T A A A A G G A G G A G T A G A A G T T C T A G A A T T A A A A C T G G G T A G $\mathrm{A}$ G C T A $T$ & 1576 \\
\hline $\mathrm{gb}|\mathrm{EF} 376992.1|$ & 1516 & T A A T A A A A G G A G G A G T A G A A G T T C T A G A A T T A A A A C T G G G T A G A T G T A T & 1567 \\
\hline $\mathrm{dbj}|\mathrm{AB} 263920.1|$ & 1516 & T A A T A A A A G G A G G A G T A G A A G T T C T A G A A T T A A A A C T G G G T A G T G C T A T & 1567 \\
\hline
\end{tabular}

Supplementary Table 1. Aligned sequences used for molecular beacon design. *, conserved

nucleotide; *, conserved nucleotide used in molecular beacon. 


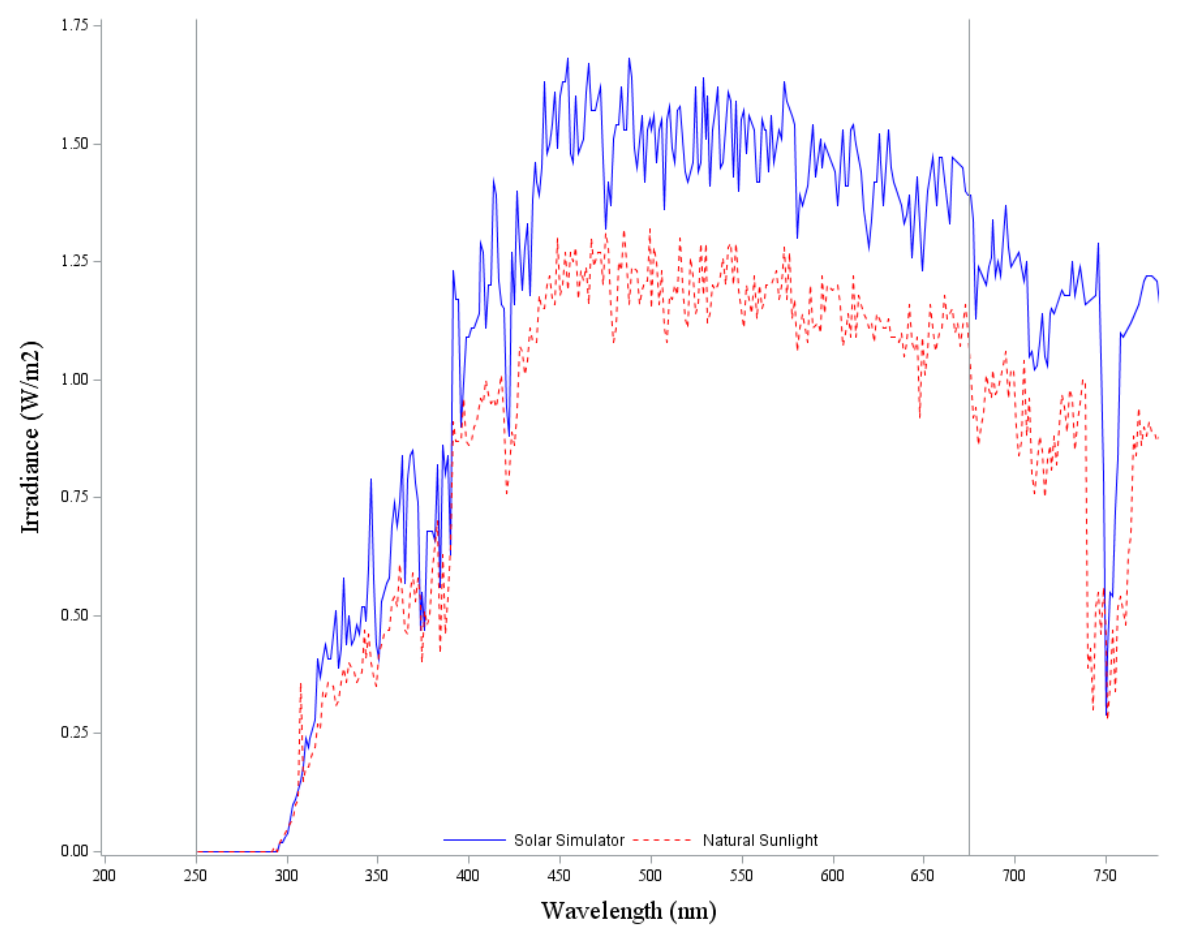

Figure S1. Irradiance of natural and simulated sunlight used in experiments. Vertical references lines placed at 250 and $675 \mathrm{~nm}$ indicate range of radiometer. Natural sunlight readings were taken on May $11^{\text {th }}, 2016$ in Riverside, CA, USA. 


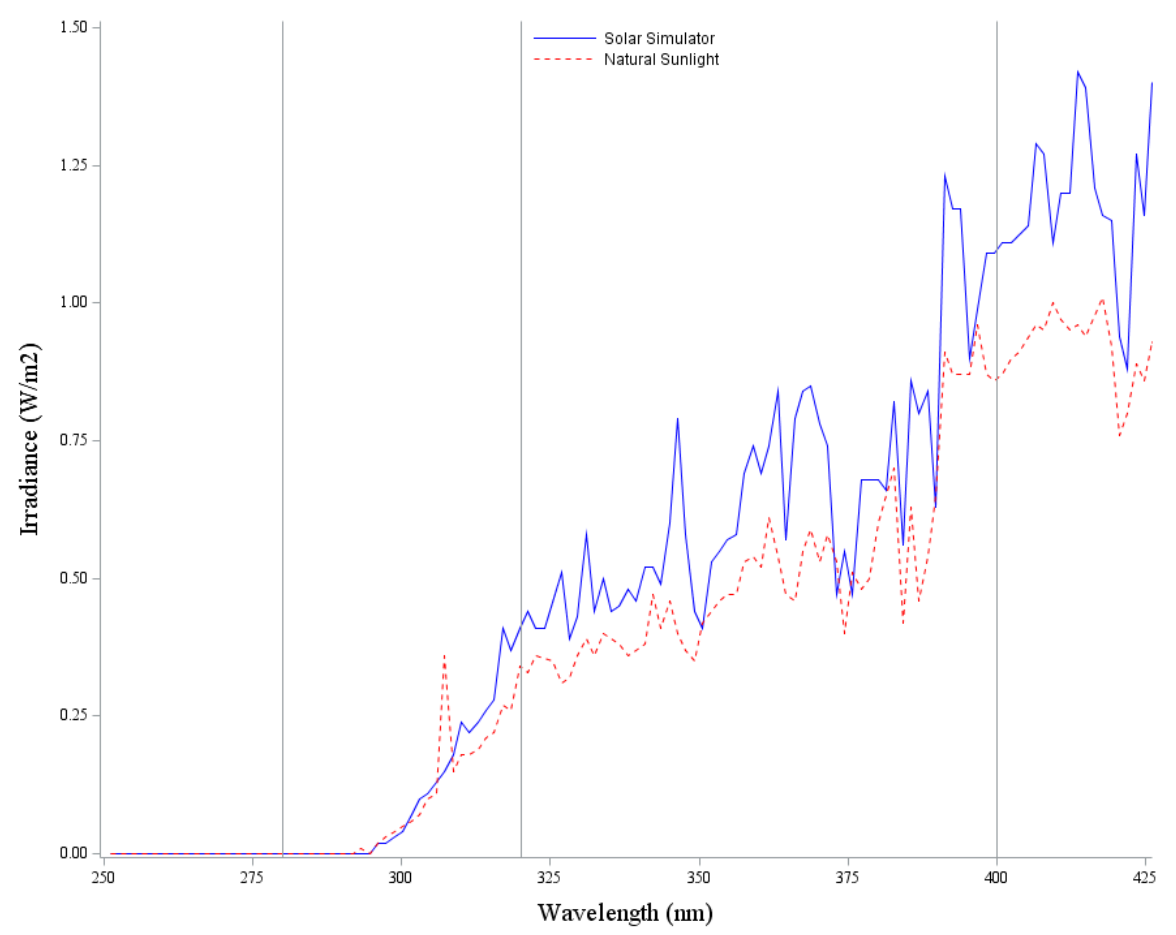

Figure S2. As above, irradiance of natural and simulated sunlight used in experiments. Vertical reference lines indicate UVB $(280-320 \mathrm{~nm})$ and UVA $(320-400 \mathrm{~nm})$ spectra.

\section{Works Cited:}

(1) Kappeler, A.; Lutz-Wallace, C.; Sapp, T.; Sidhu, M. Detection of bovine polyomavirus contamination in fetal bovine sera and modified live viral vaccines using polymerase chain reaction. Biologicals 1996, 24 (2), 131-135.

(2) Moriyama, T.; Sorokin, A. Intracellular trafficking pathway of BK Virus in human renal proximal tubular epithelial cells. Virology 2008, 371 (2), 336-349. 